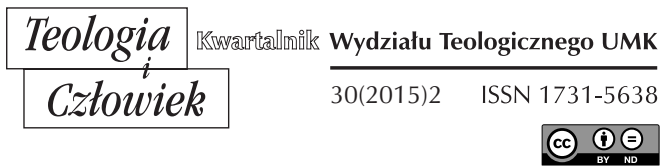

KS. RYSZARD SELEJDAK*

\title{
CURA E FORMAZIONE DELLE VOCAZIONI SACERDOTALI ADULTE
}

DOI: http://dx.doi.org/10.12775/TiCz.2015.020

\section{INTRODUZIONE}

Nella storia della Chiesa il fenomeno delle vocazioni sacerdotali che si verifica in età adulta, dopo una più o meno lunga esperienza di vita laicale e di impegno professionale, è sempre stato presente. Esso ha accompagnato la formazione presbiterale provocando problemi e costituendo qualche volta una forma di sfida verso gli ambienti e le forme tradizionali di preparazione al sacerdozio ministeriale, cioè il seminario maggiore e quello minore. Negli ultimi quaranta anni del ventesimo secolo il suo valore è diminuito in modo notevole. La maggior parte

* Ks. Ryszard Selejdak - dr hab. teologii, ur. 17.01.1958 w Kromołowie, prezbiter archidiecezji częstochowskiej, patrysta i teolog dogmatyk. Doktorat z teologii patrystycznej - 1991 r. (Papieski Instytut Patrystyczny „Augustinianum” w Rzymie), habilitacja z teologii dogmatycznej - 2011 r. (Uniwersytet Kardynała Stefana Wyszyńskiego w Warszawie). Od 15 maja 1991 r. pracownik watykańskiej Kongregacji Edukacji Katolickiej. Od 1 marca 2002 r. Dyrektor Departamentu Seminariów tej Kongregacji. Od 22 kwietnia 2013 r. Dyrektor Departamentu Seminariów watykańskiej Kongregacji ds. Duchowieństwa. 
dei seminari destinati alla formazione delle vocazioni adulte sono stati chiusi. Tuttavia, nei tempi odierni, il numero delle suddette vocazioni sta nuovamente crescendo. Perciò la cura e la formazione di esse attualmente costituisce una sfida.

\section{CONCETTO DI VOCAZIONE ADULTA}

Il concetto di vocazione adulta non ha una definizione univoca. Quando si parla di vocazioni adulte, comunemente si fa riferimento all'età: si considerano di fatto vocazioni adulte quei candidati che iniziano la preparazione al sacerdozio nell'età in cui le vocazioni "normali" ricevono gli Ordini Sacri, oppure emettono la professione religiosa perpetua. Spesso si tratta di persone che si decidono per il sacerdozio, o la vita religiosa, dopo aver compiuto interamente o in parte gli studi universitari, o dopo aver esercitato una professione o un mestiere.

Cè chi considera, in determinati casi, come primo elemento caratteristico delle vocazioni adulte non tanto letà quanto piuttosto il grado di maturità, il processo di maturazione. Situazioni che giustificherebbero questa posizione si verificano in alcune facoltà teologiche, dove non pochi studenti non sapendo decidersi per il sacerdozio rinviano la scelta vocazionale. Per altri infine, ai quali la definizione tradizionale di vocazione adulta sembra limitativa, sarebbe quella che ha prodotto un cambiamento di direzione nella scelta dello stato di vita ${ }^{1}$.

\section{VOCAZIONI ADULTE: ASPETTI, DIFFUSIONE E FORMAZIONE}

\subsection{ASPETTI}

Negli anni quaranta-settanta del ventesimo secolo, in non poche nazioni, specialmente in Germania e in Francia, sono stati eretti i seminari

${ }^{1}$ Cf. I. Peri, I Seminari. Le novità dal Vaticano II ad oggi nei documenti e nellesperienza della Chiesa, Roma 1985, p. 401-402; Id., I Seminari oggi. La formazione dei Sacerdoti nelle circostanze attuali, Roma 1995, p. 533-534. 
per le vocazioni adulte a livello sia regionale sia nazionale. Lo stimolo alla creazione di detti centri era dato soprattutto dalla diminuzione del numero delle cosiddette vocazioni "normali". Si può dire, che le vocazioni adulte sono state un vero segno dei tempi, sia per l'importanza che un po' dappertutto hanno acquisita, sia per i molteplici nuovi problemi cha hanno suscitato. $\mathrm{Da}$ un lato, rappresentavano un fenomeno che era da valutarsi positivamente, in quanto manifestavano un consolante risveglio dello spirito di apostolato nelle anime ed erano una soluzione, sia pure parziale, alla rilevante diminuzione delle vocazioni "classiche" o "normali"; di quelle vocazioni cioè che provenivano prevalentemente dai seminari minori, dai collegi ecclesiastici e dalle scuole secondarie ad indirizzo umanistico. Dall'altro lato, esse ponevano nuovi problemi di ordine pedagogico, disciplinare, strutturale, riguardanti la diversità delle vie d'accesso al sacerdozio e il pluralismo sacerdotale.

Soprattutto in alcune nazioni, per esempio in Brasile e in Francia, con i gruppi di formazione operaia, dove era vivo l'interesse per l'apostolato specifico, nella preparazione delle vocazioni adulte ci si preoccupava quasi esclusivamente dell'aspetto pastorale della formazione, senza porre la dovuta attenzione ai regolari studi filosofico-teologici. Si cercava di favorire maggiormente la capacità pastorale e l'adattamento a specifiche culture e classi sociali, e consentire una testimonianza di fede vissuta, piuttosto che di fornire una solida cultura, considerata spesso astratta e avulsa dalla vita. L'attenzione, una volta preminente, di diminuire la distanza tra le vocazioni adulte e le altre, ha lasciato il posto alla preoccupazione di raggiungere nelle prime una maturazione umana e spirituale nell'ambiente di provenienza.

Più ci si allontanava dal quadro classico della formazione, tentando nuove vie al sacerdozio, più la problematica tradizionale di ordine pedagogico e culturale cedeva il passo a problemi di ordine disciplinare, strutturale e, in ultima analisi, dottrinale: si arrivava a minare spesso l'immagine del sacerdote e la stessa essenza del sacerdozio.

In alcune regioni l'impostazione data alla formazione degli adulti ha suscitato altri gravi problemi, trattati nel Sinodo Episcopale del 1971. La situazione sembrava però avviarsi alla normalizzazione, anche grazie agli orientamenti del citato Sinodo e per i ripetuti interventi dei Sommi Pontifici. 
Mentre si osservavano comunità scolastiche e politiche laicali che tendevano ad individuare i propri candidati nei giovani, soprattutto per quanti dovevano essere formati a posti di dirigenza, nella comunità cristiana si avvertiva la tendenza a privilegiare l'accesso al sacerdozio ministeriale a persone adulte che non erano impegnate a tempo pieno nella pastorale. In tale tendenza non si calcolava forse a sufficienza il rischio delle improvvisazioni, delle indisponibilità, della non adeguata capacità culturale, della minore profondità spirituale e, di conseguenza, delle possibili incertezze circa la fede nonché del rischio di indebolire la coesione pastorale dei presbiteri diocesani.

La formazione delle vocazioni adulte, a motivo dei problemi che essi presentavano, doveva garantire alcune condizioni essenziali: unaccurata verifica dell'orientamento, congrui studi teologici e una adeguata permanenza nella comunità seminaristica, sufficiente a renderli capaci di vivere nella comunità presbiterale e diocesana. Contemporaneamente si raccomandava di rispettare la gradualità di un cammino formativo tipico di persone adulte, con una propria identità ed esperienza. Pertanto, prima ancora di avviare un esplicito cammino di formazione, era necessaria un'iniziale verifica vocazionale nella quale, per mezzo di un organico confronto con un sacerdote, dovevano essere prese in considerazione e valutate la storia personale, le motivazioni e le qualità che potevano far intuire l'idoneità della persona al ministero pastorale. Trattandosi di persone adulte, provenienti dal mondo del lavoro e della cultura, si tendeva a mantenere lopportunità che essi potessero conservare in qualche modo quei rapporti di amicizia e di solidarietà che ne avevano caratterizzata la vita precedente ${ }^{2}$.

\subsection{DIFFUSIONE}

Il fenomeno delle vocazioni adulte si è esteso particolarmente negli anni quaranta - settanta del ventesimo secolo anche a quei soggetti appartenenti a ceti che un tempo apparivano meno attenti al sacerdozio:

2 Cf. Id, I Seminari. Le novità dal Vaticano II ad oggi nei documenti e nellesperienza della Chiesa, op. cit., p. 402-404. 
gli operai, gli impiegati e, in generale, quelle persone prive o carenti di cultura umanistica.

Lattenzione riservata alle vocazioni adulte dal Concilio Vaticano $\mathrm{II}^{3}$ ha contribuito in larga misura ad approfondire studi specifici e a suscitare iniziative riguardanti il suddetto fenomeno. La diminuzione sensibile delle vocazioni "normali" era da associare alla scomparsa pressoché totale, specialmente in alcuni regioni, dei seminari minori; come anche alla crisi dei seminari maggiori. Inoltre era da mettere in conto il diffondersi di gruppi di apostolato rivolto a settori specifici, al proliferare di associazioni, di movimenti e di cosiddette comunità di base, che non nascondevano la loro preferenza per quei candidati adulti, maturati nei loro stessi ambienti.

Fattori psicologici e socio-culturali hanno fatto spesso sentire il loro influsso determinante: una certa instabilità e indecisione psicologica, spingeva i giovani ad operare scelte definitive o impegnative in età più avanzata; una maggiore sensibilità verso alcuni valori, quali la maturità di giudizio, lo spirito di iniziativa, la facilità dei rapporti interpersonali, che più facilmente si riscontravano nei giovani vissuti a diretto contatto con la vita reale, nelle diverse attività o professioni, che al contrario non si riscontravano nellambiente del seminario minore. Numerosi altri fattori determinavano e favorivano uno stato di indecisione e di timore ad assumersi, in età "non matura", obblighi gravosi di carattere permanente: la secolarizzazione progressiva di tutti i settori della vita pubblica e privata; l'influsso negativo dei mass media; le incertezze dottrinali e pastorali nei sacerdoti; i problemi di fede; le defezioni sacerdotali; la cultura erotica avversa alla vita celibataria ecc. Erano, tali, alcune delle difficoltà rilevanti che distoglievano i giovani dalla vocazione sacerdotale. Per queste ragioni, le vocazioni adulte non erano più considerate uneccezione o una soluzione di emergenza o di ripiego. Erano un fenomeno positivo da favorire, anche nel caso in cui le vocazioni "normali" erano sufficienti, purché l'attenzione riservata alle prime non conducesse alla svalutazione pratica del seminario minore o del maggiore, né ad una esaltazione unilaterale della comunità

3 Concilio Vaticano II, Decreto sulla formazione sacerdotale Optatam totius, 28 ottobre 1965, in: Enchiridion Vaticanum 1, n. 3, p. 825: "Si abbia ben cura di promuovere istituti o altre iniziative per le vocazioni adulte". 
di origine o di appartenenza, a scapito della comunità presbiterale alla quale il sacerdote apparteneva in virtù dellordine sacro ${ }^{4}$.

\subsection{FORMAZIONE}

Alle vocazioni adulte venivano riservati programmi pastorali ed educativi, che rispettavano la gradualità di un cammino formativo tipico di persone mature; ad esse veniva dato un posto ben definito nel sistema della formazione sacerdotale. La loro formazione era assicurata in seminari maggiori "regolari" - ritenuti dalla Sede Apostolica più idonei per il periodo degli studi filosofici e teologici - e in seminari "speciali", minori e maggiori.

La presenza di vocazioni adulte nei seminari maggiori "regolari" aveva raggiunto considerevoli proporzioni. Esse ponevano di conseguenza numerosi e non facili problemi educativi che mutavano la fisionomia stessa del seminario.

I seminari minori "speciali", caratterizzati dalla formazione classica, non erano sfuggiti alla crisi che aveva coinvolto i seminari minori "normali". Quelli che ancora funzionavano hanno subito trasformazioni profonde. Circa il metodo educativo, in alcuni seminari (ad esempio, nell'interdiocesano di Sankt Pölten, in Austria) si poneva maggiormente l'accento sulla formazione spirituale e su un più chiaro orientamento al sacerdozio. In altri invece (ad esempio, nei seminari tedeschi), che in precedenza non escludevano la prospettiva sacerdotale, si sono voluti impostare come i collegi e le scuole cattoliche. In essi era tenuta presente la meta del sacerdozio, ma non si richiedeva che fosse condivisa da tutti gli studenti. Questa esperienza veniva comunemente classificata come "seconda via" al sacerdozio.

I seminari "speciali" a livello filosofico-teologico si stavano invece diffondendo un po' dappertutto con buoni risultati, anche se manifestavano non pochi problemi educativi.

${ }^{4}$ Cf. I. Peri, I Seminari. Le novità dal Vaticano II ad oggi nei documenti e nellesperienza della Chiesa, op. cit., p. 404-405. 
Quasi unanimemente era riconosciuta la grande utilità di istituire per le vocazioni adulte seminari "speciali". I frutti di questo atteggiamento si possono scorgere, oltre che in Italia, in Austria, in Colombia, in Inghilterra, in Venezuela e, per certi aspetti, anche negli Stati Uniti d'America e in Spagna. In questi paesi era ritenuto valido il sistema formativo fondato sui seminari. Si era avuta la conferma che le vocazioni adulte si affermavano e progredivano dove vi era fiducia nel seminario, rinnovato secondo gli orientamenti conciliari ${ }^{5}$.

\section{ALCUNI NUOVI MODELLI DI FORMAZIONE DELLE VOCAZIONI ADULTE}

In Francia, in Germania, in Olanda, in Svizzera, in Canada ed in alcuni paesi dell'America Latina furono proposti nuovi modelli di formazione delle vocazioni adulte - con formule educative extraseminaristiche nei quali si poneva come primario il problema dell'adattamento pastorale e culturale. Si trattava di innovazioni, propizie per le sperimentazioni e le ricerche pedagogiche, in alcuni casi considerate come integrazione al sistema "classico"; in altri invece come vera e propria alternativa al seminario.

Questi modelli si potevano riassumere in tre gruppi:

a) Modello francese: la cura delle vocazioni adulte era passata dai seminari "speciali" ad una istituzione nuova, operante fuori del contesto seminaristico, chiamata "Gruppo di formazione", distinta per universitari, operai, contadini. Aveva due cicli: dopo il primo, quadriennale, i candidati passavano al seminario maggiore.

b) Modello latino-americano: adottava due metodi. Il primo, analogo a quello dei paesi europei, aveva le seguenti caratteristiche: rottura con la tradizione e scelta di orientamenti del tutto nuovi. Il metodo comportava tensione tra la fedeltà al Concilio Vaticano II, gli orientamenti della Sede Apostolica e le esigenze delle chiese particolari.

${ }^{5}$ Cf. ibidem, p. $406-407$. 
Il secondo metodo aveva come caratteristica l'adattamento culturale alle tradizioni e alle mentalità dei destinatari del messaggio evangelico. La formazione tradizionale si rivelerebbe lontana da questa esigenza.

c) Modello tedesco: costituiva la cosiddetta "terza via". Non veniva richiesto il certificato di "maturità (sia essa conseguita con studi normali - "prima via", sia con studi abbreviati - "seconda via"), il quale veniva sostituito da un esame di maturità professionale, chiamato "maturità dei dotati".

Nell'ambito del modello tedesco esistevano varie modalità, alcune delle quali costituite di quattro tappe, per un periodo complessivo di otto anni. Il carattere scientifico della formazione veniva in parte sacrificato in favore di un maggiore arricchimento dell'esperienza pastorale ${ }^{6}$.

\section{CARATTERISTCIHE DI ALCUNI SEMINARI PER LE VOCAZIONI ADULTE IN ITALIA}

In Italia il numero dei seminari sia minori che maggiori per le vocazioni adulte era più grande che in altri paesi. Essi, eretti alla fine degli anni quaranta del ventesimo secolo, agli inizi degli anni ottanta del medesimo secolo sono stati chiusi. Almeno 7 tra di essi avevano un'importanza significativa.

\subsection{SCUOLA PER LE VOCAZIONI ADULTE DELLA DIOCESI DI MILANO}

L'esperienza più significativa ed esemplare nel settore delle vocazioni adulte era offerta dalla diocesi di Milano, che fin dal 1947 aveva aperto seminario. I sacerdoti usciti da questo istituto sono stati oltre 150, pienamente fusi con gli altri sacerdoti formati nella via normale.

Tre considerazioni fondamentali presiedevano all'organizzazione del seminario.

a) E' sembrato indispensabile che le vocazioni degli adulti fossero accolte e formate in una propria sede, diversa da quella delle vocazioni "normali". Le ragioni dichiarate erano evidenti: le

${ }^{6}$ Cf. ibidem, p. $407-408$. 
vocazioni adulte avevano bisogno di orari speciali e di metodi pedagogici e didattici adeguati alla loro età e alle loro esigenze.

b) La formazione spirituale delle vocazioni adulte tendeva agli stessi traguardi delle altre vocazioni. La loro formazione intellettuale, letteraria e scientifica veniva concentrata sugli elementi essenziali, e svolta in analogia con i programmi dei corsi magistrali.

c) Era sembrato opportuno che la formazione filosofico-teologica fosse comune, e venisse data in una sede unica, sia per le vocazioni adulte sia per le "normali". Solo così si poté evitare il pericolo di creare nel clero una divisione con due differenti tipi di presbiteri ${ }^{7}$.

\subsection{SEMINARIO REGIONALE PIEMONTESE PER LE VOCAZIONI ADULTE}

Il seminario è sorto per volontà dei vescovi del Piemonte nell'ottobre 1967. La sua istituzione era stata preparata da un convegno di studio tenutosi a Torino nel settembre 1966, per iniziativa della conferenza dei superiori dei seminari della regione.

Questo centro di formazione non è sorto soltanto per aiutare i giovani carenti dal punto di vista dello studio, ma anche per permettere loro un originale e proprio cammino spirituale.

La preparazione alla teologia è stata curata in vario modo con il passare degli anni: nel primo anno veniva messa in piedi una vera e propria scuola interna privata; in seguito alcuni alunni vennero inviati come privatisti a sostenere esami di abilitazione magistrale, altri vennero inviati a frequentare la scuola magistrale di stato.

Lesperimento ebbe successo per varie ragioni, e si ritornò alla scuola di ricupero interna secondo programmi stabiliti in accordo con una commissione di professori del seminario diocesano di Torino, e con un originale metodo induttivo, ritenuto più adatto per gli adulti. Questa prassi fu sempre osservata, e l'ingresso in teologia era condizionato al superamento di un esame.

${ }^{7}$ Cf. ibidem, p. 409-410. 
L'esperienza di alcuni anni di funzionamento del Seminario Regionale Piemontese aveva suscitato delle riflessioni sul modo di concepire un seminario per le vocazioni adulte e sulla stessa "vocazione adulta". A queste riflessioni seguì la proposta che detto seminario non fosse inteso soltanto come momento di passaggio, ma come un ambiente caratteristico per la formazione ecclesiale; si ipotizzò anche quale fosse la cultura di base più idonea per la preparazione alla teologia.

Tra le note che qualificavano il seminario si possono ricordare:

- una notevole disponibilità delle vocazioni adulte all'approfondimento della preghiera;

- una libertà corresponsabile e una intensa vita comunitaria;

- uneducazione che si costruisce in continuità con l'esperienza e la cultura che ogni vocazione adulta ha acquisito precedentemente;

- un metodo di continua revisione di vita sul vangelo, sul Magistero Ecclesiastico e sulla realtà sociale in cui si era immersi;

- un diretto contatto con gli ambienti parrocchiali e associativi di origine per un più facile inserimento nell'attività pastorale.

Guardando alla precedente condizione degli alunni si notava che la grande maggioranza di questi altri proveniva dal lavoro professionale, mentre l'ambiente ecclesiale di origine era la parrocchia o i gruppi nati al suo interno. Erano meno presenti i movimenti laicali a raggio diocesano, ed erano assenti le comunità di base o di impegno sociale.

L'età, salvo alcune eccezioni, era superiore ai 24 anni al momento dell'ingresso.

Quanto alla scolarità di base, vi erano situazioni diverse: si andava dalla terza media alla laurea. Il titolo di studio più frequente era quello della scuola media superiore di tipo tecnico (geometri, ragionieri, periti...).

In seguito allesperienza formativa dei primi anni, si andava operando una più accurata selezione di quanti chiedevano di essere ammessi. Si cercava di chiarire la posizione dei singoli con contatti e colloqui con i formatori per un periodo non inferiore ad un anno ${ }^{8}$.

${ }^{8}$ Cf. ibidem, p. $410-411$. 


\subsection{SEMINARIO INTERDIOCESANO "S. CUORE", A COLLE DI VAL D'ELSA}

E' stato aperto nel 1968. Accoglieva adulti di ogni età, di ogni parte d'Italia (anche religiosi), di ogni livello culturale, classe o categoria sociale. Di fatto si andava dai marinai, agli operai, ai ferrovieri, agli artigiani, ai maestri, ai ragionieri, ai periti, ai laureati. Predominava la preparazione tecnico-scientifica; era scarsa quella umanistica.

I corsi di preparazione, che duravano massimo tre anni, prevedevano gli studi di alcune materie fondamentali di cultura generale, quali l'italiano, il latino, i principi di filosofia, e si concentravano da una parte sullo studio della "storia della salvezza" e dallaltra sulla partecipazione ampia alla liturgia di ogni giorno, con l'aggiunta di particolari pratiche di pietà.

La comunità non era chiusa in se stessa: si predisponeva in varie occasioni ad accogliere sacerdoti e laici, e i seminaristi prestavano servizio in parrocchie della diocesi, nella catechesi e nella celebrazioni liturgiche.

Affinché si potesse fare vera comunità di fede e di carità, nonostante le diversità di carattere, di cultura, di mentalità, in adulti ormai plasmati e spesso provenienti da diverse regioni d'Italia, si cercò di limitare il numero degli alunni, che non dovevano superare in totale le quindici unità.

Per i corsi filosofico-teologici, i candidati erano normalmente inviati al Pontificio Seminario Regionale di Siena. Potevano scegliere di essere alunni della loro diocesi di origine oppure di un'altra diocesi del Senese ${ }^{9}$.

\subsection{CASA "SAN GIOVANNI BATTISTA" PER LE VOCAZIONI GIOVANILI, A SAN MASSIMO-VERONA}

Era una comunità di verifica vocazionale che accoglieva giovani dai 18 anni in avanti, provenienti da varie esperienze di scuola media superiore, di lavoro o di studi universitari, che erano in seria ricerca del progetto di Dio nella loro vita e intravedevano la prospettiva della vocazione al sacerdozio ministeriale.

Nel programma formativo della suddetta Casa si cercava di realizzare tre obiettivi principali:

\footnotetext{
9 Cf. ibidem, p. 412.
} 
a) una crescita spirituale che portava il candidato a rendersi effettivamente conto se il progetto che Dio aveva di lui era la vita sacerdotale;

b) una crescita umana dal punto di vista personale e sociale; attraverso la vita in comune i giovani erano aiutati a modellare il proprio carattere, a porsi al servizio degli altri e a rispettarsi reciprocamente, accettando i limiti di ognuno;

c) uno studio che completava in modo appropriato la preparazione culturale con la prospettiva di entrare nel seminario maggiore.

Componente essenziale della Casa "San Giovanni Battista" era un'intensa vita di preghiera. Due momenti di preghiera comunitaria ne caratterizzavano la giornata: la celebrazione delle lodi il mattino e dei vespri la sera, con la celebrazione eucaristica. Il colloquio personale col Signore si realizzava durante la giornata come ognuno giudicava più consono al proprio carattere.

La comunità aveva momenti di incontro con gli alunni del seminario minore, sia nelle aule scolastiche (qualche materia era svolta in comune), sia nei tempi di svago.

Le esperienze pastorali facevano parte dell'iter formativo degli alunni.

\subsection{SCUOLA ECCLESIASTICA PER LE VOCAZIONI ADULTE (SEVA), A TRENTO}

Listituzione, fondata e diretta dalla Congregazione Sacerdotale dei Figli del Sacro Cuore di Gesù si poneva al servizio delle diocesi, offrendo ai giovani che si orientavano al sacerdozio una possibilità di formazione intellettuale e spirituale che li rendeva idonei ad affrontare, nei rispettivi seminari, gli studi filosofico-teologici.

Si accettavano aspiranti dai 17 ai 24 anni. Tuttavia si faceva qualche eccezione per i giovani con particolari doti di intelligenza, di virtù ecc.

Laspirante doveva essere presentato dal proprio vescovo, dal rettore del seminario della propria diocesi o dal parroco, con l'assicurazione scritta che in seguito sarebbe stato ricevuto nel seminario maggiore della diocesi. 
Prima dell'ingresso nell'istituto, salvo casi particolari, i superiori della SEVA credevano assai utile ci fosse un incontro personale con l'aspirante.

Gli alunni venivano ammessi in quelle classi o in quel programma di studio preordinato, in base a quanto la direzione del seminario o il corpo docente della SEVA giudicavano più corrispondente al grado di preparazione culturale dei candidati.

Per le linee della formazione globale la SEVA si riferiva ai documenti del Magistero Ecclesiastico di allora sulla formazione nei seminari.

Dall'anno scolastico 1984-1985 l'istituto intraprese una nuova fase caratterizzata da una migliore qualificazione sul piano culturale, grazie a un'equipe di docenti che, attraverso l'esperienza, stava mettendo a punto un itinerario più idoneo ad assicurare la preparazione della base culturale per accedere al seminario maggiore.

Finito il corso liceale, i candidati venivano inviati ai loro seminari per essere ammessi ai corsi filosofico-teologici. Qualora non fossero stati ricevuti per la loro scarsa preparazione culturale, ma mostravano di possedere alcune virtù umane indispensabili per il sacerdozio, la direzione della SEVA doveva cercare per essi un altro seminario e se ciò non era ancora possibile, si doveva far continuare loro gli studi, senza però fargli prendere impegni formali per l'avvenire.

Non era fissata alcuna retta mensile, ma la Congregazione Religiosa che dirigeva la scuola, vivendo di beneficenza, stabiliva con coloro che presentavano i candidati una quota minima.

\subsection{SCUOLA DI ORIENTAMENTO VOCAZIONALE - SEMINARIO, A ZEVIO}

E’ stata aperta nel 1971 dalla Congregazione Sacerdotale dei Figli del Sacro Cuore, fondata da padre Mario Venturini.

Era destinata a giovani, che desideravano di orientarsi al sacerdozio, di età superiore ai 20 anni, forniti di diploma di scuola media superiore e che hanno avuto possibilmente già un'esperienza di lavoro.

La Scuola assicurava la frequenza ai corsi dello studentato teologico di Verona. Si impegnava a conservare il contatto con il centro teologico della diocesi di provenienza del giovane per le varie iniziati- 
ve tendenti a completare la preparazione al sacerdozio. In tal modo il giovane si poteva sentire sufficientemente inserito nella propria chiesa particolare.

La direzione desiderava che il candidato prima di iniziare l'anno scolastico trascorresse qualche giorno nella Scuola, per una conoscenza reciproca e per esaminare insieme le capacità intellettuali, sociali e creative in relazione alle motivazioni della scelta.

\subsection{CORSI COMPLETI DI PREPARAZIONE PER I GIOVANI PROVENIENTI DAL MONDO OPERAIO, A FANO}

Fin dal 1967, la diocesi di Fano offriva ai giovani operai di tutte le diocesi d'Italia la possibilità di arrivare al sacerdozio, mettendo a loro disposizione un corso completo di studi che si cercava di adattare al grado della loro cultura e anche della loro particolare "forma mentis". In genere gli operai, che non hanno potuto frequentare studi regolari di scuole medie superiori, si trovavano in difficoltà nell'affrontare la teologia. L'iniziativa della diocesi di Fano si proponeva di superare questo scoglio, adottando un programma di studi adatto alle possibilità dei candidati. Questo tuttavia non doveva far pensare - affermavano gli organizzatori dei corsi - che si puntasse a livelli inferiori di cultura. Si trattava soltanto di una diversa impostazione dello studio e di una differente distribuzione nel tempo, non di un abbassamento del livello delliter formativo.

Punti fondamentali della formazione erano l'unione col vescovo, e quindi con la Chiesa; la preghiera; l'impegno nello studio; qualche forma di lavoro per il mantenimento dei candidati, almeno nello spirito, in linea di continuità con il mondo dal quale provenivano e che dovevano continuare ad amare, di un amore quasi di privilegio.

Gli ideatori dei corsi, per giustificare gli stessi corsi, oltre alle ragioni accennate, ne indicavano altre, considerate ancor più profonde ed essenziali:

a) Non si intendeva di offrire alla Chiesa sacerdoti che fossero stati da confrontare con quelli che uscivano dai seminari di tipo tradizionale. Per questo non sarebbe stato necessario - si affermava - il tentativo di una nuova istituzione, che non voleva essere un seminario di raccordo tra il mondo operaio e 
seminari comuni, ma tra il mondo operaio e il sacerdozio e, in un secondo momento, tra il sacerdozio e il mondo operaio.

b) Si voleva che quanto c'era di positivo - umanamente parlando nella personalità dell'operaio, non andasse perduto né sostituito ma, semmai, posto in evidenza, potenziato ed integrato come ricchezza da disporre nella futura attività pastorale.

c) Si voleva contribuire a creare delle nuove leve di sacerdoti che facilitassero nella Chiesa il dialogo con la mentalità del mondo operaio, riadattandone il linguaggio, e quanto altro necessario, a questa mentalità. I sacerdoti così preparati dovevano dedicarsi a un particolare tipo di apostolato: quello operaio.

I giovani che partecipavano ai corsi completi di preparazione al sacerdozio a Fano lasciavano per alcuni mesi dell'anno il lavoro, la famiglia, il loro ambiente, e decidevano di vivere in comunità per mettersi insieme in ascolto della parola di Dio, opportunamente guidati, per prendere una migliore coscienza di ciò che significava essere cristiani, di ciò che era la Chiesa e, lo stesso sacerdozio, per meglio vivere insieme l'esperienza di una comunità cristiana nella celebrazione dell'Eucaristia e nel servizio fraterno.

Si trattava di condurre i singoli candidati ad una maturità di fede, non tanto per mezzo di regole esteriori e ragionamenti umani, quanto per mezzo di una fondamentale fiducia e del credito che a loro si accordavano, lasciandoli in piena e consapevole libertà di dare una risposta a Dio che li chiamava, qualunque essa fosse. Non c'era, di conseguenza, un controllo stretto, come si poteva osservare in altri seminari.

Il progresso spirituale-formativo dei candidati doveva essere una personale conquista, e non la semplice accettazione di un disegno presentato e caldeggiato da altri, mediante "una formazione esortativa e condizionante".

In seno alla comunità ogni impegno si discuteva e si assumeva comunitariamente, dopo una esauriente maturazione.

Nel periodo della preparazione si cercava che i giovani avessero contatti con la vita degli operai. Per questo motivo si prevedeva la partecipazione degli stessi ad incontri di gruppi operai, allo studio degli aspetti e dei problemi del mondo del lavoro, alla ripresa delle attività lavorative nei mesi estivi o in alcune ore del giorno. Limpegno lavorativo doveva 
servire per assicurarsi il massimo di autonomia finanziaria, ma soprattutto per mantenere il contatto con il mondo nel quale in futuro sarebbero stati chiamati a lavorare pastoralmente.

I corsi di preparazione ebbero una durata massima di sette anni ${ }^{10}$.

\section{DIVERSITA' TRA I MODELLI ITALIANI E QUELLI DI ALTRI PAESI}

La preparazione delle vocazioni adulte in Italia si discostava notevolmente - come si può rilevare dall'impostazione dei diversi centri dalle esperienze descritte e raggruppate nei tre modelli indicati.

Il seminario maggiore, inteso nel senso del Decreto Optatam totius e della Ratio fundamentalis institutionis sacerdotalis, era considerato in genere come la base indispensabile e il punto di riferimento per ogni formazione sacerdotale, compresa quella delle vocazioni adulte.

In Italia prevaleva l'opinione che la formazione filosofica e teologica delle vocazioni adulte doveva essere curata nellambito del seminario maggiore normale, non escludendo però la necessità di proporre adattamenti speciali. Per questa ragione, i seminari speciali per le vocazioni adulte erano presi in considerazione soprattutto per il periodo degli studi secondari. I vescovi italiani, infatti, per quanto riguardava la formazione intellettuale sembravano orientarsi verso un normale curricolo filosoficoteologico, insistendo sulla necessità per i candidati di completare sempre gli studi secondari.

Infine, negli anni quaranta-settanta del ventesimo secolo si assiste al sensibile aumento delle vocazioni adulte al di fuori dei seminari speciali, le quali compievano gli studi o nel seminario maggiore o privatamente, seguendo programmi speciali. Tali casi eccezionali venivano esaminati e regolati di comune accordo tra le diocesi interessate e la Congregazione per l'Educazione Cattolica ${ }^{11}$.

${ }^{10}$ Cf. ibidem, p. 415-417.

${ }^{11}$ Cf. ibidem, p. 417-418. 


\section{LE DISPENSE DELLA CONGREGAZIONE PER L'EDUCAZIONE CATTOLICA IN FAVORE DELLE VOCAZIONI ADULTE}

Una duplice casistica veniva presentata dai vescovi alla Congregazione per l'Educazione Cattolica per le dispense in favore delle vocazioni adulte.

a) Ammissione agli Ordini Sacri di persone adulte, in genere ultraquarantenni, con titolo di studio a livello accademico, in possesso di una discreta cultura filosofico-teologica e stimate dal clero e dai fedeli, per la testimonianza di vita cristiana e per l'impegno nell'apostolato. La richiesta veniva abitualmente corredata del parere favorevole del consiglio presbiterale o del consiglio episcopale.

In conformità ad una prassi abbastanza affermata, la Congregazione concedeva al vescovo di ammettere il candidato agli Ordini Sacri, dopo averlo fatto esaminare da unapposita commissione di sacerdoti, nominati dallo stesso vescovo, per giudicare lavvenuta preparazione, che veniva fatta in genere sotto la guida di docenti di teologia o di sacerdoti esperti nel campo teologico.

La Congregazione rivolgeva una viva preghiera al vescovo affinché l'interessato, ricevuta l'ordinazione sacerdotale, continuasse l'impegno a perfezionare la formazione nel triplice aspetto: spirituale, intellettuale e pastorale, secondo le indicazioni della conferenza episcopale nazionale per la formazione permanente.

b) Ammissione agli Ordini Sacri di giovani che hanno regolarmente compiuto, con esito positivo, i prescritti studi filosoficoteologici presso istituti teologici o atenei ecclesiastici.

Questi candidati - per lo più dirigenti di movimenti e gruppi ecclesiali di giovani impegnati (quali l'Azione Cattolica, La Comunione e Liberazione, i Focolarini, il Rinnovamento nello Spirito Santo e i Gruppi catecumenali) - dal vescovo erano ritenuti idonei di ricevere gli Ordini Sacri in considerazione sia della loro preparazione spirituale, sia delle esperienze fatte in attività di apostolato, oltre che per la specifica preparazione intellettuale.

Anche in questi casi la domanda veniva abitualmente corredata del parere del consiglio presbiterale o del consiglio episcopale. 
La Congregazione per l'Educazione Cattolica autorizzava il conferimento del presbiterato alla condizione che il candidato completasse il proprio iter formativo al sacerdozio - a norma dei "motu proprio" Ministeria quaedam, riguardante i ministeri, e Ad pascendum, riguardante lordine del diaconato - passando attraverso le tappe dei ministeri e del diaconato, distanziate tra loro dagli interstizi regolamentari. Si voleva offrire all'interessato, in questa maniera, lopportunità - con la necessaria assistenza del vescovo - di acquisire progressivamente una spiritualità pastorale, di servizio e di comunione piena e sperimentata con la gerarchia ${ }^{12}$.

\section{ALCUNI DOCUMENTI DEL MAGISTERO ECCLESIASTICO RIGUARDANTI LE VOCAZIONI ADULTE}

Alcuni documenti del Magistero Ecclesiastico trattano delle vocazioni adulte, dei seminari per le vocazioni adulte e della formazione dei seminaristi in età più matura.

a) Lettera circolare Sinodi episcopali. Questioni riguardanti la formazione del clero.

Essa è stata pubblicata dalla Congregazione per l'Educazione Cattolica il 23 maggio 1968. In questo documento la Congregazione proponeva che almeno sul piano nazionale si imponessero una maggiore attenzione sulle vocazioni adulte, che una pastorale vocazionale deve permettere di scoprire, e la creazione di istituti adatti alla loro formazione sacerdotale ${ }^{13}$.

b) Lettera circolare Vocationes adultorum ai presidenti delle Conferenze Episcopali circa la cura e la formazione delle vocazioni degli adulti.

Questa Lettera è stata pure pubblicata dalla Congregazione per l'Educazione Cattolica e costituisce il documento del Magistero Ecclesiastico che tratta la suddetta problematica in modo più ampio ${ }^{14}$. Esso comprende

12 Cf. ibidem, p. 419-420.

${ }^{13}$ Cf. Congregazione per l'Educazione Cattolica, Lettera circolare Sinodi episcopali. Questioni riguardanti la formazione del clero, 23 maggio 1968, in Enchiridion Vaticanum S1, n. 203, p. 197-199.

${ }^{14}$ Cf. Id., Lettera circolare Vocationes adultorum ai presidenti delle Conferenze Episcopali circa la cura e formazione delle vocazioni degli adulti, 14 luglio 1976, in En- 
due parti: I. "Situazione attuale delle vocazioni adulte e dei seminari e prassi della S. Congregazione”, II. "Indicazioni emerse dalle deliberazioni della Assemblea Plenaria”.

In apertura si osserva: "Le vocazioni di adulti (qui prese nel senso più ampio, cioè tutte quelle vocazioni che non entrano nella nozione della cosiddetta vocazione o via educativa «normale») in questi ultimi anni sono state molto numerose in tutta la Chiesa. Sono da considerarsi veramente in modo positivo come segno di un consolante rinnovamento spirituale nelle assemblee di uomini maturi e rimedio per attenuare almeno in parte lodierna penuria di vocazioni sacerdotali" 15 .

La Lettera sottolinea che nonostante le esperienze generalmente buone avute nei seminari con vocazioni adulte è necessaria "una maggiore prudenza e circospezione nella selezione di tali vocazioni" e di "una certa pedagogia adattata alle esigenze delletà o della condizione socio-culturale dei singoli candidati". Le idee circa la loro formazione differiscono nelle diverse nazioni e "danno spazio in varia misura al pluralismo pedagogico e sacerdotale" 16 .

In seguito la Lettera osserva che in alcune nazioni per vari motivi si è orientato a risolvere i problemi pedagogici delle vocazioni adulte nell'ambito del seminario normale. Tale soluzione non di rado sembra offrire vari vantaggi ai seminari stessi, sia per l'aumento degli alunni sia per le nuove esperienze spirituali e apostoliche con le quali gli adulti possono giovare agli altri compagni di corso. Tuttavia in casi di tal genere di solito si deve insistere sullosservanza delle norme canoniche quanto alla durata del corso di studi, evitando le riduzioni arbitrarie del corso filosofico e teologico, senza la dovuta dispensa ${ }^{17}$.

Il documento successivamente difende l'esistenza dei seminari minori contro l'esaltazione esagerata delle vocazioni degli adulti, ricordando che "lodierna penuria di vocazioni non può essere superata con le sole vocazioni di adulti [...]. Dobbiamo qui notare anche una nociva propensione che oggi si diffonde in alcune regioni tendente a scindere

chiridion Vaticanum 5 (1974-1976), n. 2097, p. 1373; F. Marchisano, La Santa Sede e la formazione del clero, in: Lateranum LVI (1990) 2, p. 623-624.

15 Lettera circolare Vocationes adultorum, n. 2098, p. 1373-1375.

16 Ibidem, n. 2098-2099, p. 1375.

17 Cf. ibidem, n. 2099, p. 1375. 
dalla «istituzione» la cura delle vocazioni e la formazione sacerdotale per trasferirle alla «base», alla quale vengono dati il diritto e la facoltà di formare i sacerdoti per le proprie esigenze particolari secondo criteri speciali propri. La qual cosa non è ammissibile. Infatti la formazione sacerdotale, e di conseguenza anche la figura del sacerdote, devono dipendere dalla volontà della legittima autorità ecclesiastica, alla quale soltanto compete il diritto di regolamentare la formazione, la vita e l'azione del sacerdote con opportune leggi e norme"18.

La Congregazione ribadisce che molte questioni controverse della condizione sacerdotale (il lavoro manuale o professionale rimunerato, la "professionalizzazione" dei sacerdoti, il ministero a tempo pieno o limitato, la lotta di classe, l'attività politica, il celibato) trovano "terreno" favorevole nelle "piccole comunità" del Brasile e nei "gruppi di formazione" in Francia, Canada e Belgio, i quali pretendono di proporre un nuovo modello di sacerdote e in conseguenza anche un nuovo tipo di formazione sacerdotale. "Queste nuove formule pedagogiche generalmente insistono sulla necessità di riconoscere pieno valore formativo agli elementi insiti nella cultura popolare e alle concrete esperienze della vita; elementi che in talune circostanze vengono preferiti alla formazione intellettuale classica. Tuttavia, benché non si possa negare l'utilità di tali esperienze, è molto difficile accettarle in qualità di prima condizione, quasi generale ed esclusiva, per accedere al sacerdozio come qua e là si pretende. La Sacra Congregazione ritiene che si debbano sconsigliare i vari esperimenti di tal genere, soprattutto quando sono proposti in situazioni agitate e confuse e pretendono semplicemente di sostituirsi alla formula educativa classica [...]. Nella formazione delle vocazioni adulte di qualunque genere e di qualunque grado culturale si deve dare importanza fondamentale ad una solida formazione spirituale e teologica. Infatti questa deve essere integra nella sostanza e certa e sana nella dottrina; non va confusa con la formazione diaconale o con la preparazione dottrinale dei laici all'apostolato [...]. Nel formare le vocazioni adulte si deve usare la massima attenzione per custodire l'unità del sacerdozio cattolico. Perciò nell'attuare i vari adattamenti necessari non si devono accentuare le differenze che

${ }^{18}$ Ibidem, n. 2100, p. 1377. 
separano gli "adulti" dalla via normale; al contrario si deve fare in modo che questa distanza in qualche modo venga ridotta o con opportuni mezzi compensata"19.

Quest'ultima osservazione è stata in seguito ancora una volta ribadita nel testo. Essa esprime sollecitudine e preoccupazione per l'unità del clero e per la sua coesione interna nella diocesi, contro la tentazione di dar vita a due classi di sacerdoti, "distinti dal punto di vista della cultura e della legge del celibato". Il documento ricorda alle Chiese locali la necessità di mostrare più attenzione nei confronti delle vocazioni degli adulti, nonostante abbiano un numero sufficiente di vocazioni "classiche", ad esse preferite ${ }^{20}$.

La seconda parte della Lettera circolare Vocationes adultorum comprende le osservazioni e le indicazioni dei padri dell'Assemblea Plenaria della Congregazione per l'Educazione Cattolica emerse dalla descrizione della realtà fatta nella prima parte del documento. Si osserva che le vocazioni adulte presentano una buona qualità spirituale e una buona idoneità pastorale, e "manifestano il desiderio di una vita impegnata e austera nell'ambito di un seminario bene strutturato quanto a disciplina, studi e direzione spirituale. Fra i padri prevalse il parere della necessità di curare la formazione filosofica e teologica delle vocazioni adulte nel seminario maggiore normale, considerando sempre tuttavia l'opportunità di speciali adattamenti per i singoli casi. Ne consegue la necessità o l'opportunità di avere seminari per le vocazioni adulte per gli studi medi (ginnasio e liceo) più che per gli studi filosofico-teologici. Soltanto pochi padri sembravano espressamente favorevoli alla formula di un seminario maggiore per vocazioni adulte. Da ciò si può dedurre che la creazione di tali seminari non si può raccomandare indiscriminatamente, ma va piuttosto saggiamente esaminata e vagliata nei singoli casi, secondo le particolari circostanze del luogo" 21 .

Tra le mancanze nel campo della preparazione scientifica si constatano la scarsa preparazione umanistica e filosofica e le difficoltà nello

${ }^{19}$ Ibidem, n. 2101-2102, p. 1379-1381.

${ }^{20}$ Cf. A. Orczyk, Il rinnovamento della formazione seminaristica, Roma 1997, p. 94.

${ }^{21}$ Lettera circolare Vocationes adultorum, n. 2105-2106, p. 1383. 
studio del latino. Secondo la maggior parte dei padri "si deve richiedere da tutti i candidati almeno una conoscenza minima di questa lingua" 22 .

In questa parte della Lettera risulta pure che alcuni padri "hanno riconosciuto la necessità di istituire particolari sperimentazioni pedagogiche con le vocazioni adulte in determinate situazioni e circostanze (per es. in taluni gruppi sociali, o regioni del cosiddetto «Terzo Mondo», ecc.). Tali sperimentazioni tuttavia si dovranno condurre sotto la responsabilità delle conferenze episcopali e con l'approvazione della Congregazione per l'Educazione Cattolica"23.

Infine, nella Lettera della Congregazione per l'Educazione Cattolica, sono formulate alcune proposte concrete che coinvolgono soprattutto questo Dicastero. "All'ufficio per i seminari è stata affidata l'opportunità di uno studio più approfondito dei problemi riguardanti l'adattamento pedagogico e culturale, che oggi pare doversi richiedere in talune situazioni particolari. A tale scopo è stato deciso che presso la Congregazione per l'Educazione Cattolica si costituisca una speciale commissione composta da rappresentanti della medesima S. Congregazione e delle Congregazioni per l'Evangelizzazione dei Popoli, per i Religiosi e gli Istituti Secolari. Inoltre, all'ufficio per i seminari è stato affidato l'incarico di continuare a raccogliere elementi più abbondanti non solo circa l'esistenza e il numero delle vocazioni adulte, ma anche circa la loro qualità e attitudini pastorali, quali si manifestano nel ministero pastorale dopo l'ordinazione sacerdotale"24.

La Congregazione deve "dedicare particolare attenzione alla formazione spirituale delle vocazioni adulte, soprattutto di quelle che seguono speciali vie di preparazione", alla loro formazione teologica, e deve "vigilare affinché le assidue cure delle vocazioni adulte non avvengano a danno del seminario maggiore, diminuendo in pratica l'importanza e la stima e insinuando nell'animo uneccessiva esaltazione della comunità di origine o di adesione a danno della comunità presbiterale, alla quale il sacerdote appartiene in forza del sacramento" 25 .

\footnotetext{
${ }^{22}$ Ibidem, n. 2106, p. 1383.

${ }^{23}$ Ibidem.

${ }^{24}$ Ibidem, n. 2108, p. 1385.

${ }^{25}$ Ibidem.
} 
c) Codice di Diritto Canonico (1983)

Codice di Diritto Canonico fa riferimento alle vocazioni adulte nel can. $233 \$ 2$ in cui stabilisce che "I sacerdoti e soprattutto i Vescovi diocesani si impegnino perché coloro che in età più matura si ritengono chiamati ai ministeri sacri siano prudentemente aiutati con la parola e con l'opera e preparati nel debito modo".

d) Ratio fundamentalis institutionis sacerdotalis

Il documento pubblicato dalla Congregazione per l'Educazione Cattolica in versione aggiornata il 19 marzo 1985 propone di erigere istituti formativi e di adottare metodi adeguati per la formazione delle vocazioni adulte: "Secondo le necessità di ciascuna nazione si erigano e si favoriscano istituti destinati alla formazione di coloro che sono chiamati al sacerdozio in età più avanzata. Queste particolari case di formazione sacerdotale, con l'aiuto dei vescovi della regione o anche della nazione, siano configurate e ordinate in modo tale da poter corrispondere pienamente al loro scopo.

E' necessario che tali istituti abbiano un proprio regolamento per la pietà, la disciplina e gli studi, affinché gli alunni di età più matura

- tenuto conto delleducazione ricevuta da ciascuno precedentemente,

- mediante un opportuno metodo pedagogico e didattico possano ricevere la formazione spirituale e scientifica che loro manca, e che si stimi necessaria per iniziare gli studi ecclesiastici.

Tenuto conto delle circostanze locali, si dovrà pure giudicare se questi alunni, dopo avere con sufficiente spazio di tempo compiuti gli studi medi, siano in grado di continuare i corsi ordinari dei seminari, oppure debbano frequentare speciali scuole filosofiche e teologiche"26.

e) Esortazione Apostolica Pastores dabo vobis

Lultimo documento del Magistero Ecclesiastico che fa riferimento al fenomeno delle vocazioni adulte e la loro formazione è l'Esortazione Apostolica Pastores dabo vobis di Giovanni Paolo II. A tale riguardo il Pontefice scrive: "Come è sempre avvenuto nella storia della Chiesa, e

${ }^{26}$ Congregazione per l'Educazione Cattolica, Ratio fundamentalis institutionis sacerdotalis, 19 marzo 1985, n. 19, in: Congregazione per l'Educazione Cattolica, Documenti (1969-1989). Formazione dei sacerdoti nel mondo d’oggi, Roma 1990, p. 489-490. 
con qualche caratteristica di confortante novità e frequenza nelle attuali circostanze, va registrato il fenomeno di vocazioni sacerdotali che si verificano in età adulta dopo una più o meno lunga esperienza di vita laicale e di impegno professionale. Non è sempre possibile, e spesso non è neppure conveniente, invitare gli adulti a seguire l'itinerario educativo del Seminario maggiore. Si deve piuttosto provvedere, dopo un accurato discernimento dellautenticità di queste vocazioni, a programmare una qualche forma specifica di accompagnamento formativo così da assicurare, mediante opportuni adattamenti, la necessaria formazione spirituale e intellettuale. Un giusto rapporto con gli altri candidati al sacerdozio e periodi di presenza nella comunità del Seminario maggiore potranno garantire il pieno inserimento di queste vocazioni nell'unico presbiterio e la loro intima e cordiale comunione con esso" ${ }^{27}$.

\section{SEMINARIO NAZIONALE POLACCO PER LE VOCAZIONI ADULTE}

Il 9 settembre 2014 la Congregazione per il Clero, ha eretto il Seminario Nazionale Polacco per le Vocazioni Adulte con l'approvazione del suo Statuto, a norma del can. $237 \$ 2$ del C.I.C. La suddetta approvazione è stata fatta ad experimentum per un periodo di tre anni.

Lo Statuto del sopraccitato Seminario, che definisce la sua identità giuridica e pedagogica, è composto da 4 pagine ed è suddiviso in Introduzione, 7 capitoli e 36 paragrafi.

Nell'Introduzione si menziona che uno dei "segni di oggi" nella Chiesa polacca è l'aumento del numero dei candidati al sacerdozio in età adulta. I Vescovi, essendo responsabili della promozione delle vocazioni al sacerdozio e per la loro formazione, hanno deciso di istituire un Seminario per le Vocazioni Adulte che garantirebbe la formazione sacerdotale dei candidati di età compresa tra i 36-60 anni. La formazione in questo Seminario abbraccia le quattro dimensioni fondamentali: umana, spirituale, intellettuale e pastorale.

${ }^{27}$ Giovanni Paolo II, Esortazione Apostolica Pastores dabo vobis, 25 marzo 1992, n. 64. 
Nel capitolo primo intitolato Costituzione, natura e fine del Seminario, si dichiara, tra l'altro, che il Seminario Polacco per le Vocazioni Adulte è interdiocesano ed è destinato per la formazione dei candidati al sacerdozio in età adulta provenienti da tutte le diocesi polacche di rito latino ${ }^{28}$. La formazione sacerdotale in esso si svolge secondo le prescrizioni dei documenti del Magistero Ecclesiastico, della Ratio institutionis sacerdotalis polacca, del presente Statuto ed i relativi Regolamenti ${ }^{29}$. La sede del Seminario si trova in Cracovia. Per questo motivo, l'Arcivescovo di Cracovia, come delegato della Conferenza Episcopale Polacca, esercita una diretta supervisione e tutela del Seminario, pur nel rispetto delle competenze della Conferenza Episcopale Polacca ${ }^{30}$. I diaconi e i presbiteri ordinati lavoreranno nelle diocesi di origine ${ }^{31}$.

Il capitolo secondo Direzione del Seminario, stabilisce che la Conferenza Episcopale esercita una supervisione sul Seminario tramite il suo delegato che è l'Arcivescovo di Cracovia ${ }^{32}$. La medesima Conferenza: nomina il Rettore del Seminario o lo revoca durante il suo mandato; approva la Relazione annuale riguardante l'andamento del Seminario; cura il funzionamento economico del Seminario; modifica lo Statuto, dopo aver ricevuto il nihil obstat da parte della Congregazione per il Clero; approva il Regolamento del Seminario e le sue modifiche; decide dell'estinzione del Seminario e della devoluzione dei suoi beni ${ }^{33}$.

Nel capitolo terzo, che porta il titolo Equipé dei formatori, lo Statuto menziona il gruppo dei formatori del Seminario. Esso è composto dal Rettore, dal Padre Spirituale, dal Prefetto e dall'Economo. Il Rettore è nominato dalla Conferenza Episcopale Polacca per una cadenza di cinque anni, finita la quale, può essere nominato per una seconda, ma ultima. Altri formatori vengono nominati dall'Arcivescovo di Cracovia. Lo Statuto in questo capitolo descrive anche le incombenze dei formatori ${ }^{34}$.

\footnotetext{
${ }^{28}$ Cf. Statuto del Seminario Nazionale Polacco per le Vocazioni Adulte, par. 2.

${ }^{29}$ Cf. ibidem, par. 4.

${ }^{30}$ Cf. ibidem, par. 5.

31 Cf. ibidem, par. 6.

${ }^{32}$ Cf. ibidem, par. 7.

${ }^{33}$ Cf. ibidem, par. 8.

${ }^{34}$ Cf. ibidem, par. 9-16.
} 
Il capitolo quarto intitolato Candidati descrive il profilo del seminarista del suddetto Seminario. Egli è di solito un uomo da 35 anni in sù, celibe (scapolo o vedovo), con il diploma di maturità o di qualche studio superiore. Una speciale verifica è prevista per gli uomini con prole, che hanno ottenuto lannullamento del matrimonio, che sono già stati seminaristi e coloro che hanno oltre 60 anni di età. Gli aspiranti vengono mandati al Seminario dai singoli Vescovi diocesani, dopo aver raccolto i pareri dei sacerdoti competenti (in particolare del parroco). La decisione di un eventuale allontanamento dell'aspirante dal Seminario viene presa dal Vescovo che l'ha mandato dopo aver consultato il Rettore. Lammissione allordinazione viene decisa dal Vescovo, tenendo conto del parere del rettore e dei superiori ${ }^{35}$.

Nel capitolo quinto Formazione seminaristica viene presentata la formazione dei futuri sacerdoti nel suddetto Seminario. Essa abbraccia la dimensione umana, spirituale, intellettuale e pastorale. E' strutturata nell'arco di un quinquennio (biennio filosofico e un triennio teologico) e viene preceduta da un anno propedeutico, che serve soprattutto al discernimento della vocazione e all'introduzione alla vita spirituale ${ }^{36}$. Per quanto riguarda la formazione intellettuale viene prescritto che essa si svolga nell'ambito dello Studium domesticum organizzato sulla base di un accordo con la Facoltà di Teologia. Gli aspiranti all'ordinazione sacerdotale, prima dell'ammissione al presbiterato, devono superare un esame dellintero programma di teologia, ma non sono obbligati ad ottenere il titolo accademico di magister ${ }^{37}$.

Il capitolo sesto con il titolo Organizzazione e mantenimento del Seminario, dichiara che dell'ubicazione della sede del Seminario e del suo mantenimento economico decide la Conferenza Episcopale Polacca in accordo con il Vescovo diocesano del luogo dove si trova il Seminario ${ }^{38}$. Il Seminario è sostenuto materialmente dalle Diocesi di provenienza dei seminaristi oppure da loro stessi, se hanno tale possibilità. Norme parti-

\footnotetext{
${ }^{35}$ Cf. ibidem, par. 17-23.

${ }^{36}$ Cf. ibidem, par. 24-27.

37 Cf. ibidem, par. 28.

38 Cf. ibidem, par. 31.
} 
colari a tale riguardo saranno elaborate sotto la direzione dell'Arcivescovo di Cracovia ${ }^{39}$.

Nel capitolo settimo intitolato Prescrizioni finali si dichiara che lo Statuto presente si approva ad experimentum per tre anni ${ }^{40}$. Esso può essere modificato da parte della Conferenza Episcopale Polacca in seguito allottenuto nihil obstat da parte della Congregazione per il Clero ${ }^{41}$. Il Seminario può essere chiuso per gravi cause sulla base del decreto della Conferenza Episcopale Polacca ${ }^{42}$. Nel caso dellestinzione del Seminario i suoi beni saranno devoluti alla Conferenza Episcopale Polacca ${ }^{43}$.

All'inizio dell'anno accademico 2014-2015 nel Seminario Nazionale Polacco per le Vocazioni Adulte in Cracovia hanno cominciato la formazione al sacerdozio 9 candidati.

\section{CONCLUSIONE}

Il fenomeno delle vocazioni sacerdotali che si verificano in età adulta nella Chiesa è un vero segno dei tempi, sia per l'importanza che un po' dappertutto ha acquistato, sia per i molteplici nuovi problemi che suscita. Da un lato, le suddette vocazioni rappresentano un fenomeno che è da valutarsi positivamente in quanto manifestavano un consolante risveglio dello spirito di apostolato nelle anime. Infatti sono un rimedio, sia pure parziale, alla rilevante diminuzione delle vocazioni "classiche" o "normali" che prevalentemente provengono dalle scuole secondarie a indirizzo umanistico. Dallaltro lato, esse pongono nuovi problemi di ordine pedagogico, disciplinare, strutturale, riguardanti l'identità sacerdotale e la preparazione al sacerdozio. I Vescovi e tutti quelli che hanno a cuore il bene delle vocazioni adulte, si impegnano in diversi modi a promuoverle e adeguatamente formarle. Dato che non sempre è possibile, e spesso neppure conveniente, invitare gli adulti a seguire l'itinerario educativo del Seminario Maggiore, si provvede, dopo un accurato discernimento dell'au-

\footnotetext{
${ }^{39}$ Cf. ibidem, par. 32.

${ }^{40}$ Cf. ibidem, par. 33.

${ }^{41}$ Cf. ibidem, par. 34.

${ }^{42}$ Cf. ibidem, par. 35.

${ }^{43}$ Cf. ibidem, par. 36.
} 
tenticità di queste vocazioni, a programmare una qualche forma specifica di accompagnamento formativo così da assicurare, mediante opportuni adattamenti, soprattutto una solida formazione spirituale ed intellettuale. Infatti questa deve essere integra nella sostanza, certa e sana nella dottrina, nonché non confusa con quella dei diaconi permanenti o dei laici. Inoltre nella formazione delle vocazioni adulte si deve dedicare una massima attenzione per custodire l'unità del sacerdozio cattolico per non dar vita a due classi di sacerdoti, distinti dal punto di vista della cultura e della legge del celibato. Perciò nell'attuare i vari adattamenti necessari si deve evitare l'accentuazione delle differenze che separano gli "adulti" dalla via normale; al contrario si deve fare in modo che questa distanza in qualche modo sia ridotta o compensata con opportuni mezzi.

Streszczenie: Powołania kapłańskie odkrywane w dojrzałym wieku - troska o nie i ich formacja. W historii Kościoła fenomen powołań kapłańskich odkrywanych w dojrzałym wieku był zawsze obecny. Stanowił on jeden z bardzo ważnych „znaków czasu", zarówno ze względu na duże znaczenie, jakie zyskał w wielu kościołach lokalnych, jak również ze względu na różnorodne nowe problemy, które spowodował. Z jednej strony, powołania te były pozytywnie oceniane jako przejaw owocności apostolstwa powołaniowego i jako w pewnym stopniu środek zaradczy na znaczący spadek powołań „klasycznych” („normalnych”), wywodzących się w większości ze szkół średnich o profilu humanistycznym. Z drugiej strony powołania kapłańskie odkrywane w dojrzałym wieku przyczyniły się do powstania wielu problemów o charakterze pedagogicznym, dyscyplinarnym i strukturalnym, dotyczących tożsamości kapłaństwa służebnego oraz przygotowania do niego. Różne doświadczenia kościołów lokalnych pokazują, iż biskupi i ci wszyscy, którym leżało na sercu dobro tych powołań, nie szczędzili sił, aby je promować i zagwarantować im właściwą formację. W sposób szczególny dbano, aby kandydaci mogli dogłębnie rozeznać autentyczność powołania do kapłaństwa oraz aby zapewnić im solidną formację duchową i intelektualną. Troszczono się też, aby formacja powołań kapłańskich odkrywanych w dojrzałym wieku była oparta na wizji kapłaństwa służebnego zgodnej z obowiązującą doktryną Kościoła oraz by różniła się od formacji diakonów stałych i osób świeckich zaangażowanych w duszpasterstwie. Ponadto w formacji wielką uwagę poświęcano zachowaniu jedności kapłaństwa służebnego. Istniało bowiem realne niebezpieczeństwo stworzenia dwóch rodzajów kapłanów, różniących się między sobą poziomem przygotowania oraz sposobem rozumienia i przeżywania celibatu.

Słowa kluczowe: powołania kapłańskie odkrywane w dojrzałym wieku; powołania „klasyczne” lub „normalne”; czynniki psychologiczne i społeczno-kulturowe; duszpasterstwo powołaniowe; rozeznanie powołania; modele formacji powołań kapłańskich 
odkrywanych w dojrzałym wieku; formacja duchowa i intelektualna; celibat; seminaria; jedność kapłaństwa służebnego; Magisterium Kościoła; Kongregacja.

Summary. Care and formation of delayed vocations. In the history of the Church, the phenomenon of priestly vocations coming from those who have already lived as adults is a constant feature. This has served as a real sign of the times, whether through the importance it has acquired in many local churches, or through the many new issues, which have emerged. On one hand, these so-called delayed vocations have represented a phenomenon which has been judged very positively, insofar as they served as a sign of the vitality of vocation work, resulting in at least a partial remedy for the considerable diminution of "classic" ("normal") vocations, who predominantly come from humanities oriented secondary schools. On the other hand, delayed vocations present new pedagogical, disciplinary, and structural challenges regarding both priestly identity and preparation for the priesthood. The varied experiences of local churches show that Bishops, and all who have at heart the promotion of delayed vocations, must make efforts in various ways to promote them and adequately form them. In a particular way, they must ensure a deeper discernment of the authenticity of these vocations, and their solid spiritual and intellectual formation. The entire formation process must be founded upon a vision of priestly ministry which is fully in conformity with the doctrine of the applicable Ecclesiastical Magisterium, and which also must be differentiated from the formation received by candidates for the permanent diaconate, or by those lay people who undertake works of the apostolate. Great attention must be paid so that, in the formation of delayed vocations, the oneness of the Catholic priesthood is protected. The impression should never be given that there are two classes of priesthood, distinct from the cultural point of view and regarding the law of celibacy.

Keywords: Delayed vocations; classic or normal vocations; psychological and socio-cultural factors; vocation formation; vocation discernment; models for the formation of delayed vocations; spiritual and intellectual formation; seminary; celibacy; oneness of the ministerial priesthood; Ecclesiastical Magisterium; Congregation. 
